\title{
Research Paper: \\ The Effectiveness of Premarital Counseling Based on a Relationship Education Pro- gram on the Idealistic Expectations of Single Young Adults
}

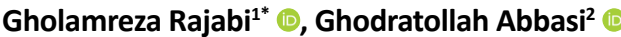

1. Department of Counseling, Shahid Chamran University of Ahvaz, Ahvaz, Iran

2. Department of Psychology, Sari Branch, Islamic Azad University, Sari, Iran.

\begin{tabular}{|c|c|}
\hline $\begin{array}{l}\text { Use your device to scan } \\
\text { and read the article online }\end{array}$ & ditation Rajabi G, \& Abbasi G. [The Effectiveness of Premarital Counseling Based on a Relationship Education Program \\
\hline arpisin & $\begin{array}{l}\text { on the Idealistic Expectations of Single Young Adults (Persian)].Iranian Journal of Psychiatry and Clinical Psychology. 2020; } \\
\text { 25(4):384-395. http://dx.doi.org/10.32598/ijpcp.25.4.3 }\end{array}$ \\
\hline 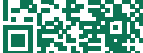 & dol http://dx.doi.org/10.32598/ijpcp.25.4.3 \\
\hline
\end{tabular}

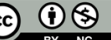

Received: 10 May 2019 Accepted: 18 Sep 2019 Available Online: 01 Jan 2020

Key words: Premarital counseling, Relationship education program, Idealistic expectations

\begin{abstract}
A B STRACT
Objectives This study aimed to examine the effectiveness of premarital counseling based on a relationship education program in reducing the idealistic marital expectation in single young adults of Ahvaz city. Methods The is a quasi-experimental study with pretest, posttest, and follow-up design using control group. Thirty two single young adults were voluntarily participated and assigned randomly into intervention group $(n=16)$ and control group $(n=16)$. The data were collected using Jones and Nelson's Marital Expectation Scale (idealistic expectation subscale) at three stages: pre-test, post-test, and 60-day followup. The intervention group received relationship education program in 9 sessions, twice a week for 90 minutes, while the control group received premarital counseling only in one session. Data were analyzed using ANOVA with repeated measures in SPSS V. 21.

Results The premarital counseling based on the relationship education program could reduce the idealistic marital expectation in the intervention group from pretest stage to the follow-up stage.

Conclusion The relationship education program can be used as a useful and appropriate program in premarital counseling and in reducing the idealistic expectations of marriage in young people.
\end{abstract}

\section{Extended Abstract}

\section{Introduction}

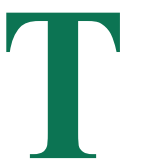

oday, expectations are an extremely important issue for marriage research. Couples have expectations from marriage that result from interactions between them and a number of factors, such as family, beliefs, traditions, and etc. When people meet each other to create a relationship, they consciously or unconsciously discuss about their expectations for making a coherent story [1]. Studies have shown that one of the most dangerous factors that ruins marital satisfaction and its stability is the irrational, idealis- tic and romantic expectations and beliefs of people from marriage [2, 3]. Most scholars conceptualize the marital expectation as a rational and cognitive process [6]. Azzopardi believes that marital expectations are the conscious and unconscious senses, concepts, ideals, illusions, desires, hopes and dreams that a person has about marriage [1]. People who begin their marriage with an extreme idealistic image of their partner and relationship are more prone to experiencing acute frustration than those with less idealistic expectations. Their relationships begin with a debilitating decline, ignorance of problems, and being superficial, which pushing couples towards frustration and potentially divorce [8].

\section{* Corresponding Author}

Gholamreza Rajabi, PhD.

Address: Department of Counseling, Shahid Chamran University of Ahvaz, Ahvaz, Iran

Tel: +98 (61) 33331366

E-mail: rajabireza@scu.ac.ir 
The review of literature on premarital counseling showed that it is one way to change or reduce idealistic expectations and beliefs about marriage [14, 15]. Premarital counseling is an important prevention strategy for families and individuals to avoid the negative consequences of getting divorce [16, 17]. Findings suggest that premarital counseling can lead to improved and increased realistic expectations, reduced pessimistic expectations [11] reduced idealistic expectations [3], individual growth of single girls [20], improved social skills and self-efficacy of girls on the brink of marriage.

One of the programs that is used in premarital counseling is the relationship education program. The target of relationship education program is young people who may be engaged in an intimate relationship. These programs can play an important role in promoting the realistic marital expectations, having appropriate attitude towards marriage, and increasing the optimism about healthy relations and communication skills in young people, especially those who may have a negative attitude towards marriage due to personal experience or history of parental divorce [24].

Relationship education program has been developed in various forms for different age groups such as school students, soldiers, university students and young people who are about to get married in order to develop their skills for having healthy relationships with others. This program can be taught in a classroom as part of a seminar in weekly workshops or by individual [25]. Gardner et al. showed that a relationship education program can positively affect people's knowledge, behavior, and attitudes towards marriage [27]. Adler-Baeder et al. also found that participants of relationship education programs have a more rational or realistic attitude towards marriage and relationships [23]. Grimm indicated that this program leads to significant changes in the attitudes and beliefs of students [5]. Johnson indicated that the young adults who participated in marriage education classes have more optimism about relationships and more realistic attitudes about marriage [29]. The study aimed to examine the effectiveness of premarital counseling based on a relationship education program on reducing the idealistic marital expectation of single young adults.

\section{Method}

The is a quasi-experimental study with pretest, posttest, and extended follow-up design using the control group. Participants were 32 single young adults in Ahvaz, Iran whose scores of the idealistic expectation dimension in Jones and Nelson's Marital Expectation Scale (MES) [31] were higher than the median value and had met inclusion and exclusion criteria. They were assigned randomly into two groups of intervention $(n=16)$ and control $(n=16)$. All relationship education sessions were conducted from October to February, 2018 in Ravan Asa Consulting Center (affiliated to the Psychology and Counseling Organization of the Islamic Republic of Iran) in Ahvaz city. The intervention group received relationship education program in 9 sessions, twice a week for 90 minutes, and the control group received one session of premarital counseling only. All groups were assessed before test, after test, and at 60-day follow-up period.

The MES instrument has 40 items and three realistic, idealistic, and pessimistic dimensions. The items are rated on a 5-point Likert scale from $1=$ Strongly disagree to $5=$ Strongly agree. In this study, we used only the idealistic dimension which has 14 item [30]. The Cronbach's alpha coefficient for MES has been reported 0.80 [30] and 0.79 [12]; and it has a significant correlation with romanticism and love styles [30]. Nilforushan et al. using confirmatory factor analysis, confirmed that the MES has three factors (realistic, idealistic, and pessimistic expectations). The Cronbach's alpha of this scale was reported 0.83 in males, 0.82 in females and 0.83 in the whole sample [31]. Collected data were analyzed using ANOVA with repeated measures in SPSS V. 21.

\section{Results}

As shown in Table 1, the $\mathrm{F}$ ratio for the group effect was significant on idealistic expectation $(\mathrm{F}=113.17, \mathrm{P}<0.001)$ which indicates that the intervention with relationship education program was effective in reducing the idealistic expectation of marriage in the intervention group compared to the control group (43.53 Vs. 55.58).

The $\mathrm{F}$ ratio for the measurement stage effect was also significant on idealistic expectation $(\mathrm{F}=11.47, \mathrm{P}<0.001)$. This

Table 1. ANOVA results of group effect on the idealistic expectation of marriage (between-group)

\begin{tabular}{cccccc}
\hline Source & Sum of Squares & df & Mean Square & F & P \\
\hline Group & 3492.09 & 1 & 3492.09 & 113.17 & $<0.0001$ \\
Error & 925.64 & 30 & 30.85 & & \\
\hline
\end{tabular}


Table 2. ANOVA results of measurement stage effect on the idealistic expectation of marriage (within-group)

\begin{tabular}{cccccc}
\hline Source & Sum of Squares & df & Mean Square & F & P \\
\hline Measurement stage & 626.02 & 2 & 313.01 & 11.47 & $<0.0001$ \\
Group $\times$ stage & 622.93 & 2 & 313.46 & 11.41 & $<0.0001$ \\
Error & 1637.04 & 60 & 27.28 & & \\
\hline
\end{tabular}

indicates a significant difference between the three stages of pre-test, post-test, and follow-up in the idealistic marital expectation (Table 2). Moreover, the $\mathrm{F}$ ratio for measurement stage effect in the intervention group was also significant $[\mathrm{F}=17.48, \mathrm{P}<0.001]$. Benfroni follow-up test was used to determine exactly in which of the three stages in the intervention group, the reduction in idealistic expectation was significantly different. Comparisons showed a significant difference between pre-test and post-test stages (12.12) and between pre-test and follow-up stages (8.62) in reduced idealistic expectation of marriage. However, there was no significant difference between post-test and follow-up stages (-3.50). Furthermore, there was a significant interaction effect of group and stages on the reduction of idealistic expectation $(\mathrm{P}<0.001)$

\section{Conclusion}

The purpose of this study was to investigate the effectiveness of premarital counseling along with a relationship education program in reducing the idealistic expectations of marriage in single young people. The results revealed that the education program was able to somewhat reduce the idealistic marital expectations of participants. This is consistent with the results of other similar studies $[3,11,23,25,27$, 28]. It can be said that the relationship education program including five components of self-knowledge, knowledge of love and dating, knowledge of happy marriage, knowledge of sexual differences, and knowledge of intimacy was effective which, in turn, can lead to the reduction of idealistic marital expectations [23].

The first step in choosing a life partner is self-knowledge and self-awareness. The answer to the question 'whom should I marry?' depends on knowing himself/herself. People who are getting married usually emphasize on knowing the partner. Since they cannot know themselves, they cannot find out who they want or do not want [2]. In this regard, one of the components of relationship education program is selfknowledge which can be a possible reason for reducing the idealistic expectations of subjects in the intervention group compared to the control group. In this component, people get to know their family of origin including attachment styles. Based on the attachment theory, parent's influence and attachment to the primary caregiver or attachment styles can play an important role in the expectations of individuals towards marriage [24]. Another possible reason for reduced idealistic expectations of marriage in the intervention group can be the knowledge of sexual differences. Gender and related issues are one of the factors that influence the development of attitudes, beliefs and expectations in a marriage [24]. Lin and Raghubir [34] showed that when realistic attitudes towards marriage are taught, people are more willing to modify their own unrealistic expectations. In our study, the given animated presentations of sexual differences and its impact on the lifestyle could lead to a reduction in the idealistic expectations of subjects.

\section{Ethical Considerations}

\section{Compliance with ethical guidelines}

This article has a code of ethics with the number 371/9 from Shahid Chamran University of Ahvaz. In addition, in the implementation of this research, ethical considerations such as the free will to participate in research, informed consent, and confidentiality of information have been observed.

\section{Funding}

This study did not receive any specific grant from funding agencies in the public, commercial, or not-for-profit sectors.

\section{Authors contributions}

All authors contributed equally in preparing all parts of the research

\section{Conflicts of interest}

The authors declared no conflicts of interest. 


\title{
اثربخشى آموزش قبل از ازدواج به شيوه برنامه آموزش ارتباط بر كاهش انتظارات ايدهآل تَرايانه جوانان مجرد أمون
}

\author{
"غلامرضا رجبى' هـ قدرتاله عباسى ' \\ 1. كروه مشاوره، علوم تربيتى و روانشناسى، دانشكاه شهيد جمران اهوازء، اهواز، خوزستان، ايران.

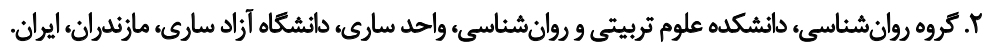

\begin{abstract}
حكيد

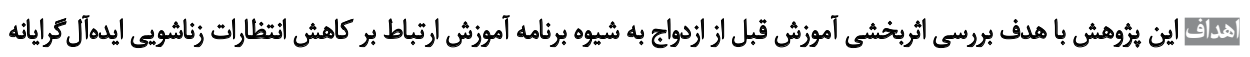
جوانان مجرد شهر اهواز انجام كرفت.

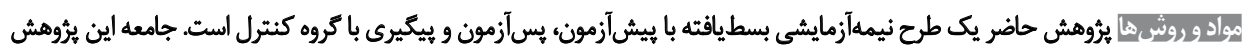

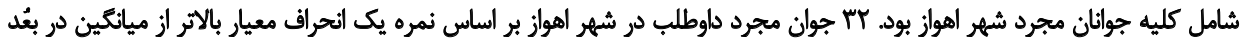

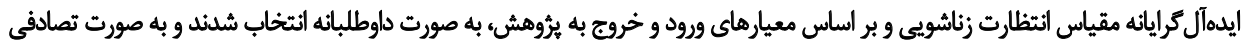

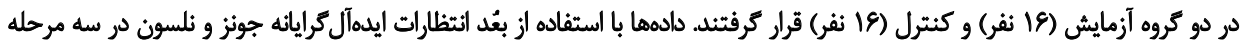

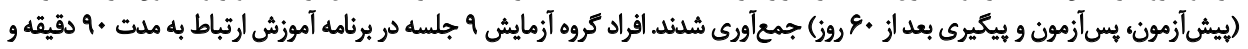

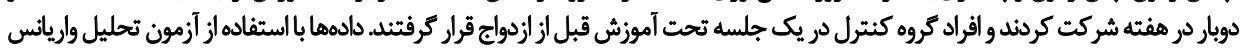

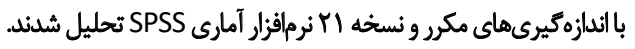

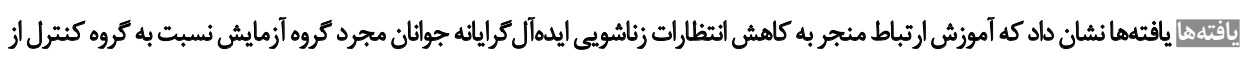

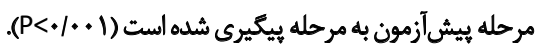

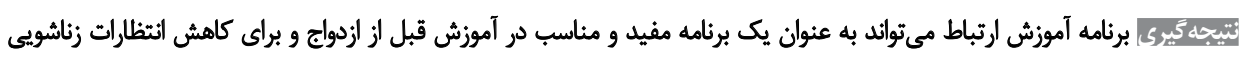

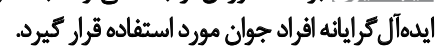

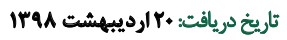

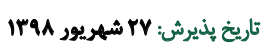

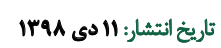

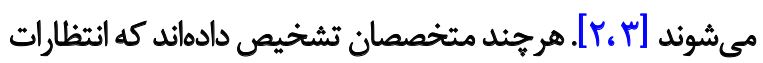
dalo

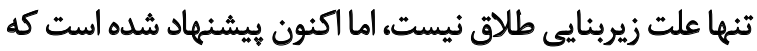

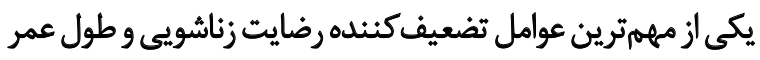

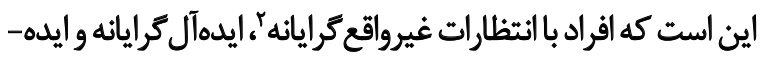

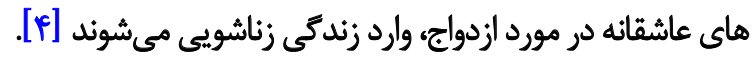

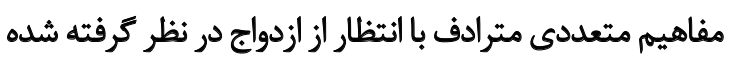

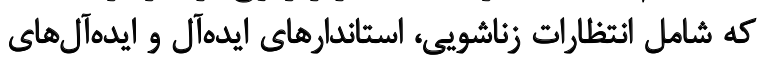

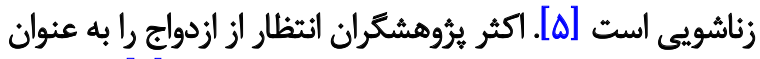

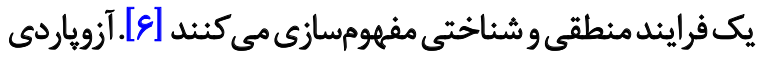

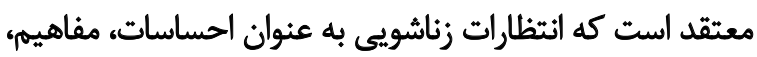

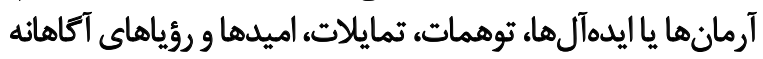

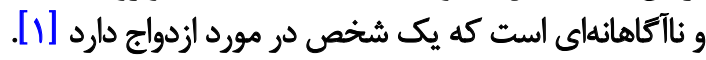

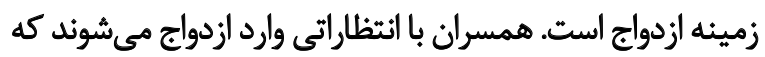

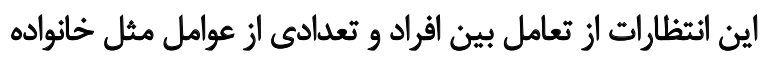

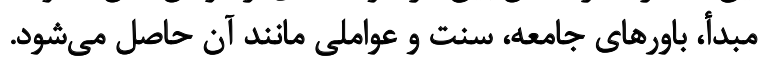

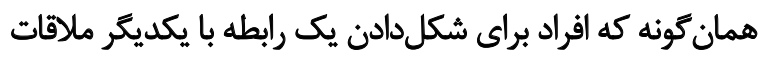

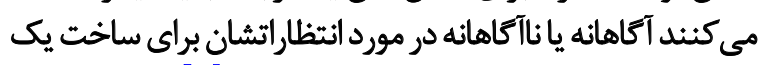

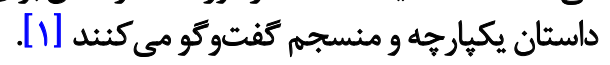

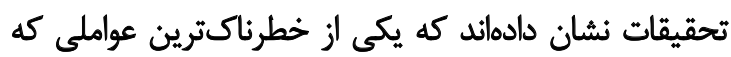

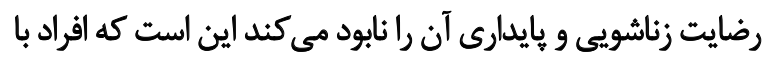

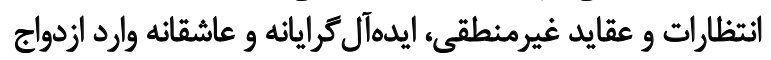

1. Expectation

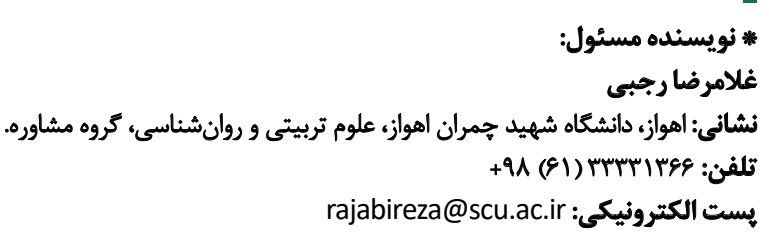




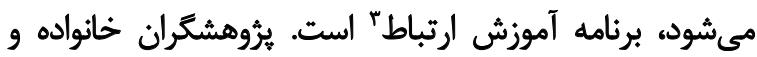

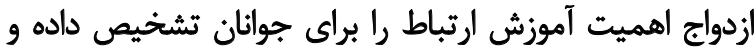

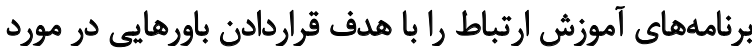

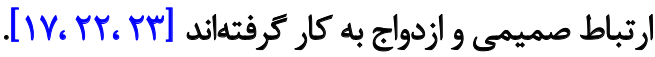

هدف برنامههاي آموزش ارتباط، افراد جوان هستئد كه ممكن

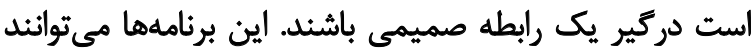

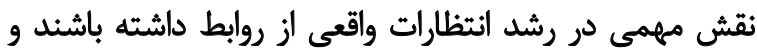

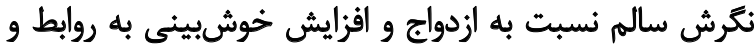

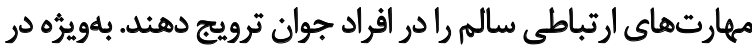

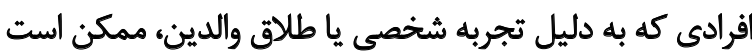

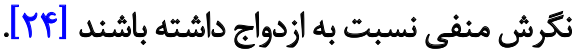

آموزش ارتباط در شكلهاى مختلفى براى ردههاى سنى مثل

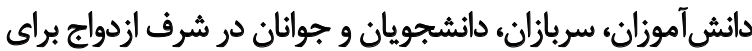

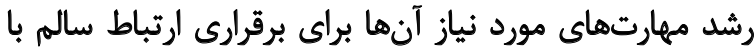

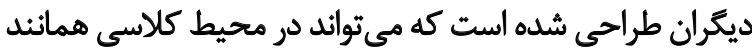

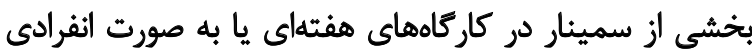

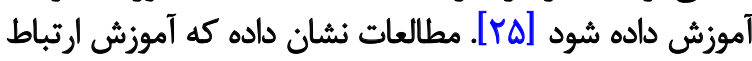

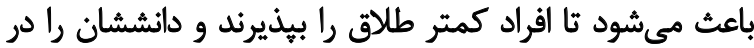

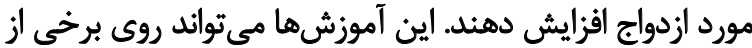

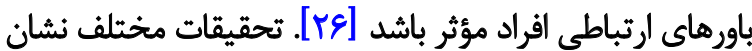

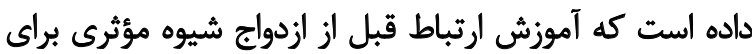

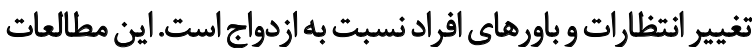

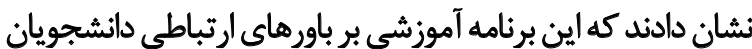

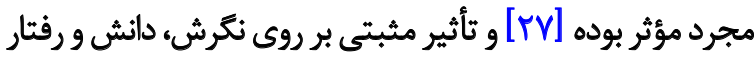

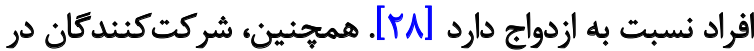

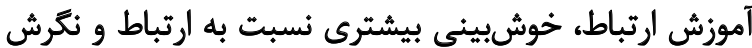

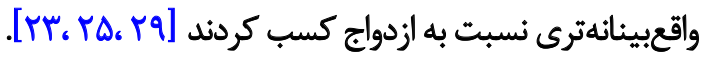

در اين ئزوهش، به دليل فقدان برنامه آموزشى مناسب براى

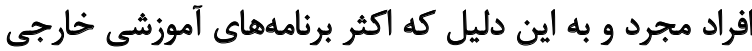

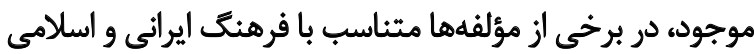

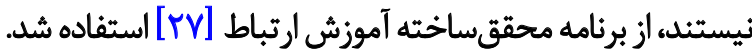

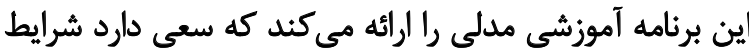

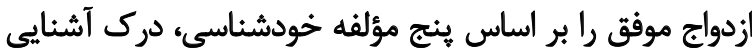

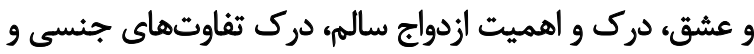

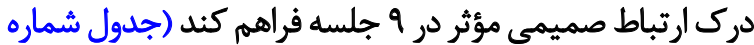

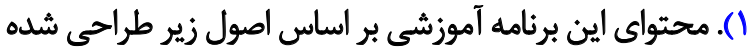

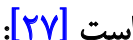

1. علم ويشكيرى: علم بيشكيرى به طور خاص به دنبال

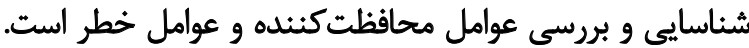

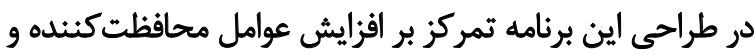

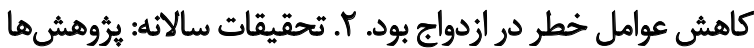

انتظارات به عنوان تصاويرى از ازدواج تعريف مى شود كه زن رئ

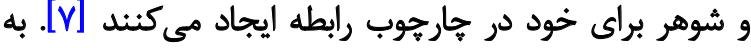

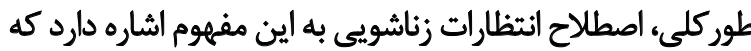

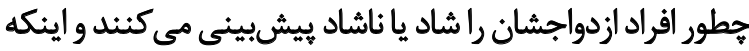
جقيدر احتمال مى دهند كه طلاق بكيرند. افرادى كه اززدواجشان را با يك تصوير ايدهآل كراى افراطى

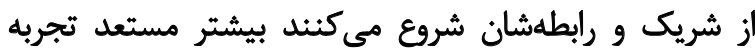

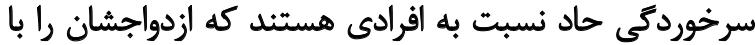

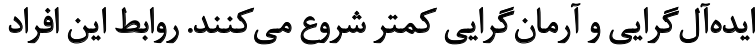

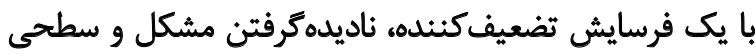

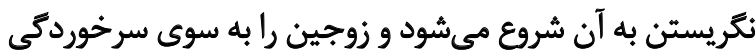

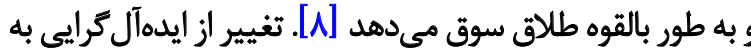

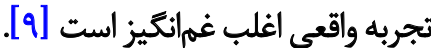
آرمانكرايى در مورد ازدواج ممكن است در رشد انتظارات

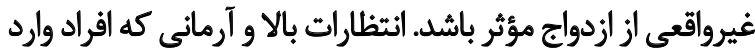

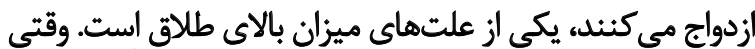

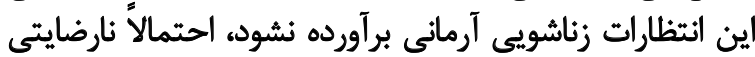

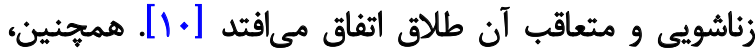
افرادى كه انتظارات زناشويى بالا و آرمان مرايانه دارئن إنداند رضايت

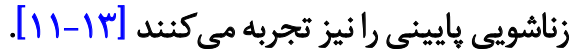

به هر حال، بيشكيرى بسيار مؤثرتر از ثلاش براى اصلاح

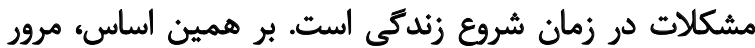

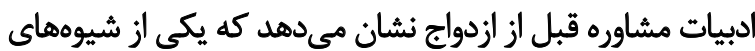

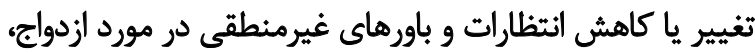

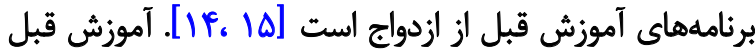

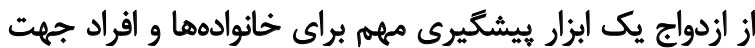

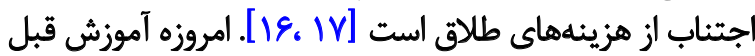

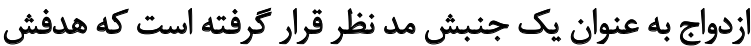

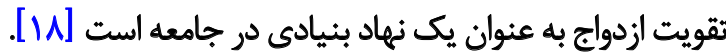
مركز ملى تحقيقات ازدواج سالم بيان مى كند كه آمادكى قبل

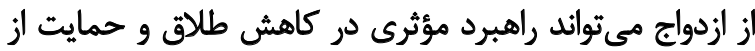

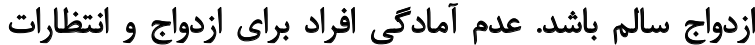

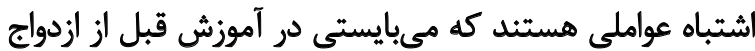

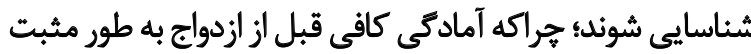

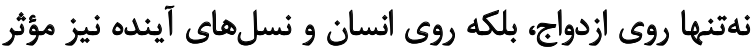

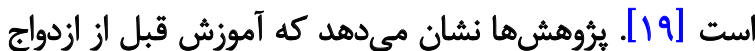

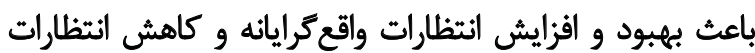

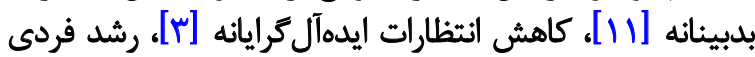

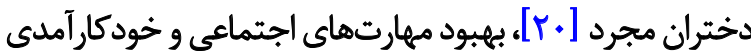
دختران در آستانه ازدواج [إب] شده است.

يكى از برنامههايى كه در زمينه آموزش قبل از ازدواج استفاده 


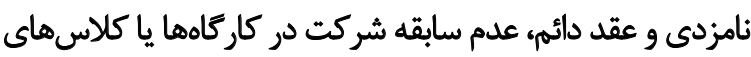

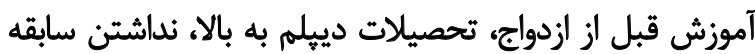

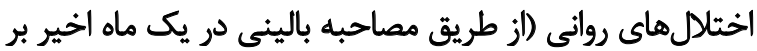

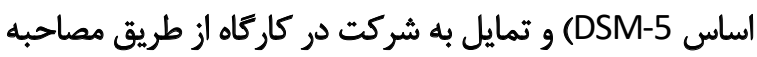

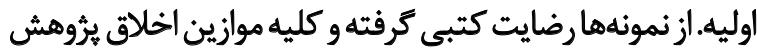

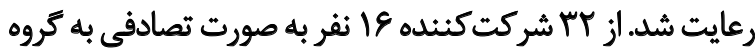

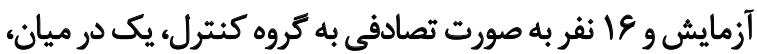

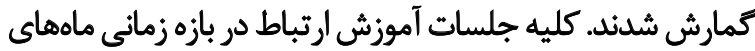

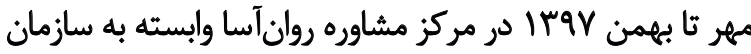

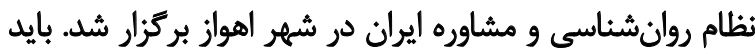

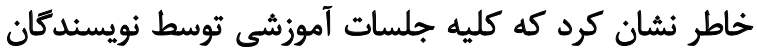

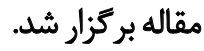

در آغاز به مدت يك ساعت هدف اين نوع آموزش و شركت برت

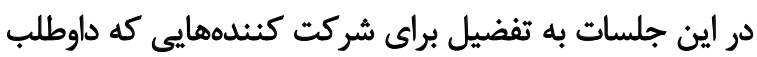

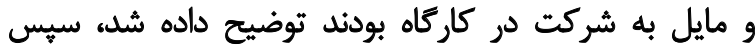

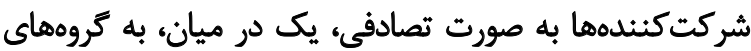

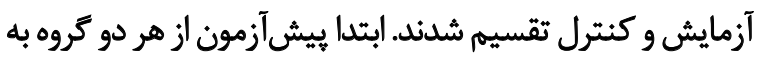

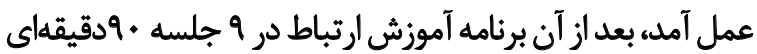

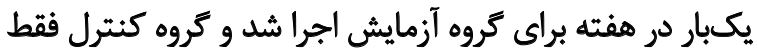

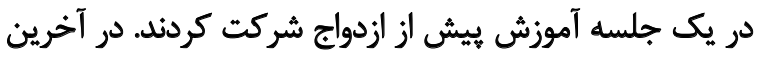

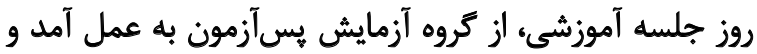

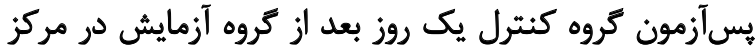

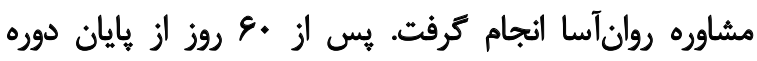

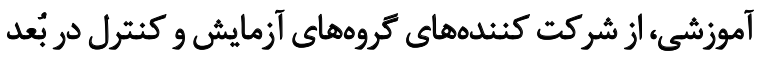

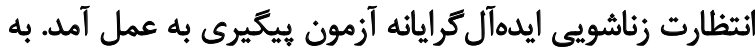

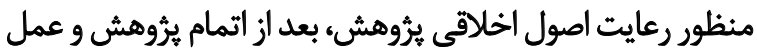

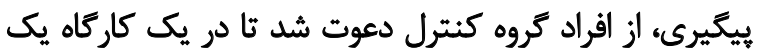

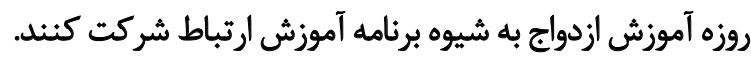
براى اندازهيرى انتظارات زناشويى از مقياس انتظارات

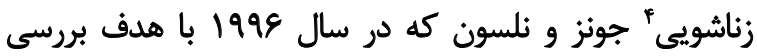

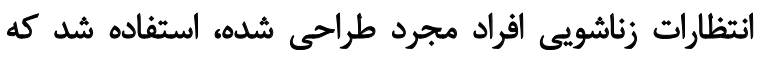

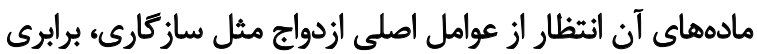

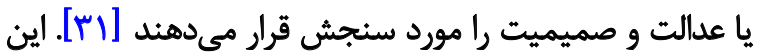

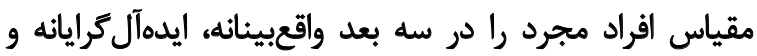

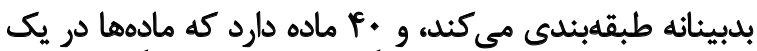

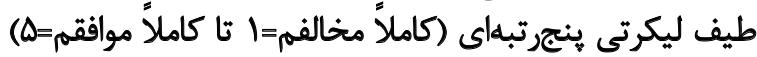

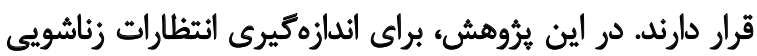
فقط از بعد مقياس أ امادهاي انتظارات ايدهآل كرايانه استفاده شد.

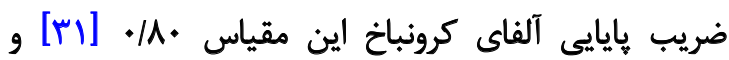

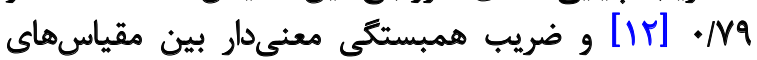
رمانتيك كرايى، نكرش نسبت به عشق بامقياس انتظارات زناشويى مئي

4. The Marriage Expectation Scale
در زمينه عشق، آشنايى، انتخاب همسر و رضايت زناشويى نشان

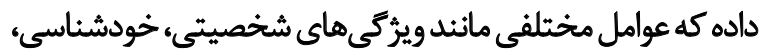

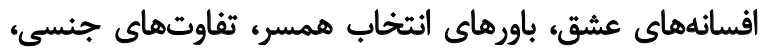

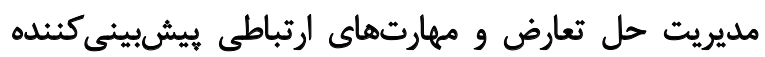

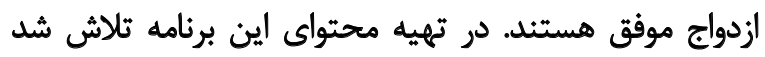

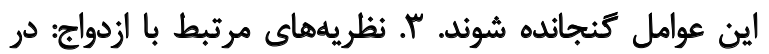

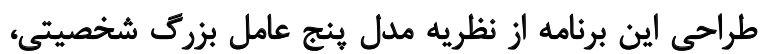

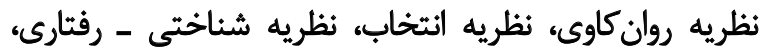

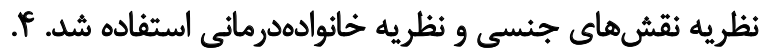

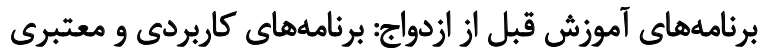

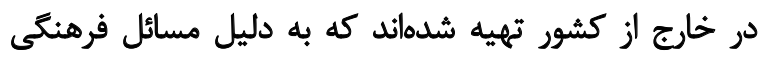

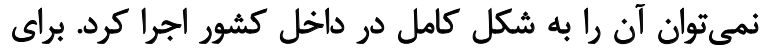

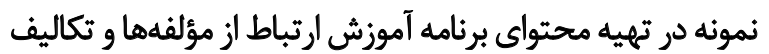

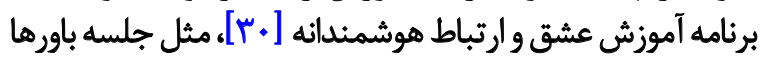

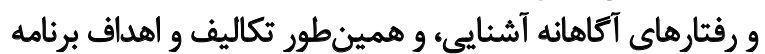
آموزش ارتباط صميمى [? [r] استفاده شد.

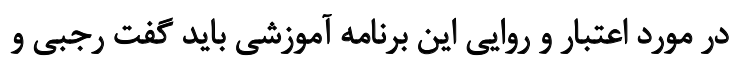

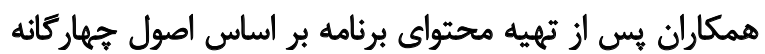

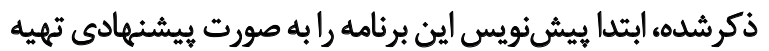

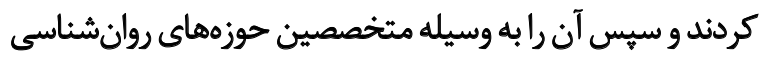

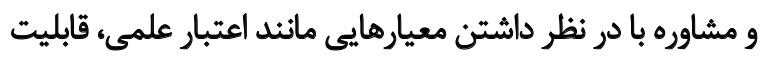

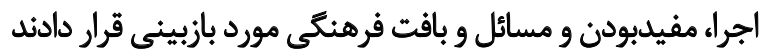

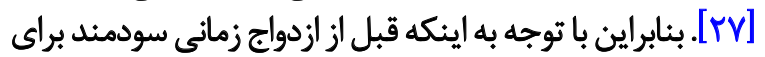

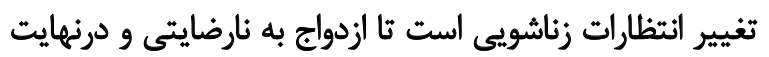

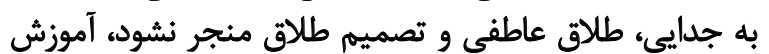

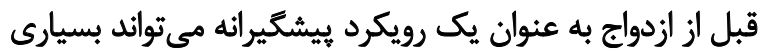

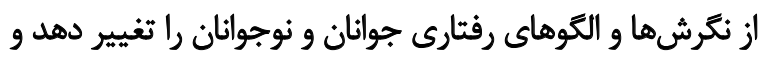

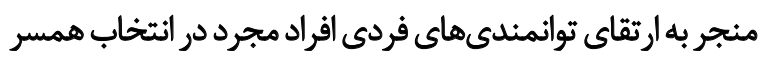

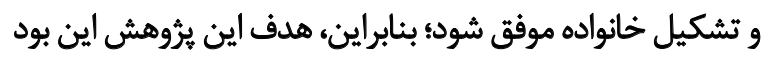

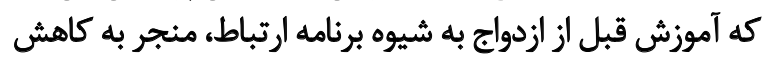
انتظارات ايدهآل كرايانه جوانان مجرد شود بهود

ووشي

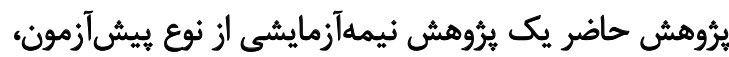

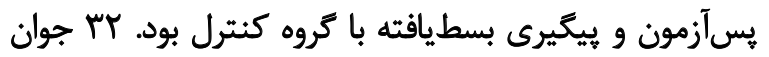

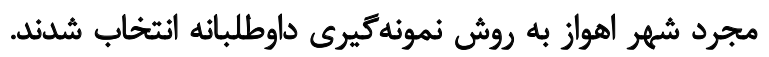

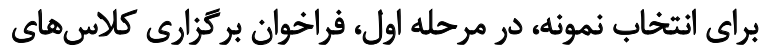

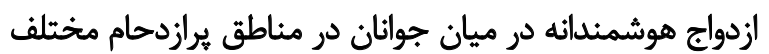

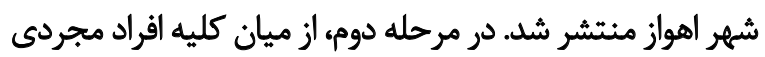

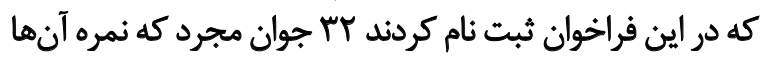

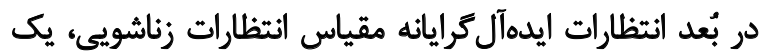

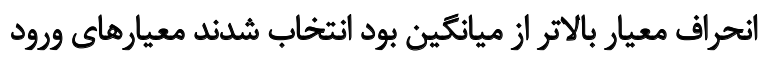

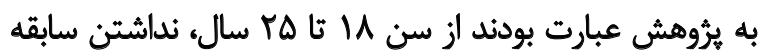


جدول ا. صورت جلسههاى آموزش قبل از ازدواج مبتنى بر برنامه آموزش ارتباط

\begin{tabular}{|c|c|}
\hline محتواى جلسه & جلسه \\
\hline 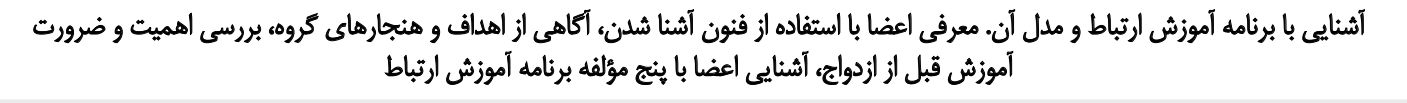 & اول \\
\hline 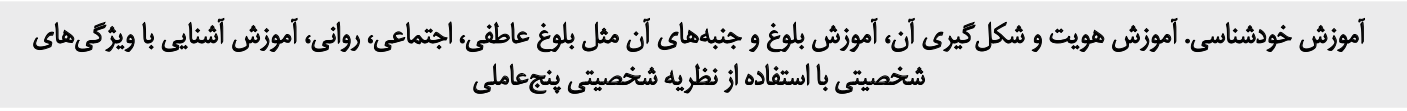 & دوم \\
\hline آموزش خودشناسى. آكاهى اعضا نسبت به سبكهاى دلبستكى، سبكهاى ارتباطى، شناسايى ينج نياز اساسى در اعضا بر اساس نظريه كالاسر و نقش & سوم \\
\hline 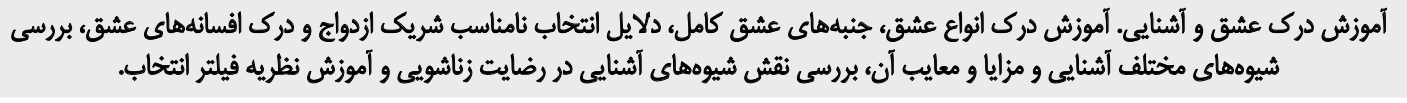 & جهارم \\
\hline 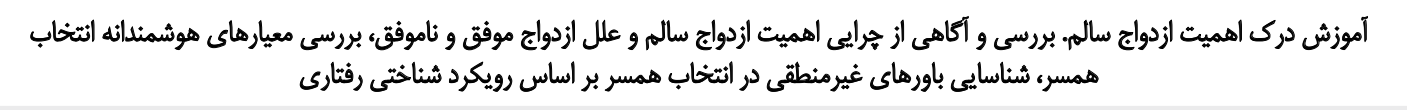 & ينجم \\
\hline 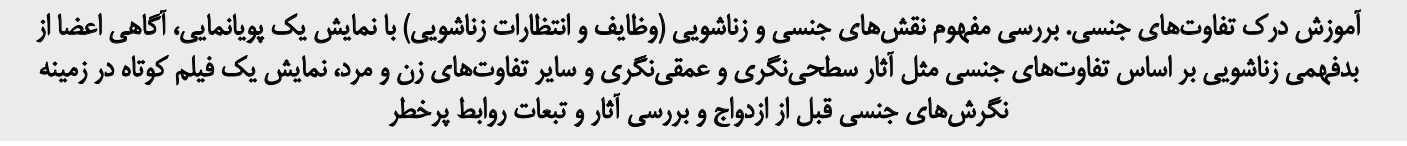 & ششم \\
\hline 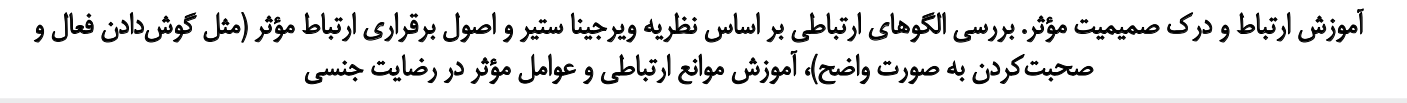 & هفتم \\
\hline 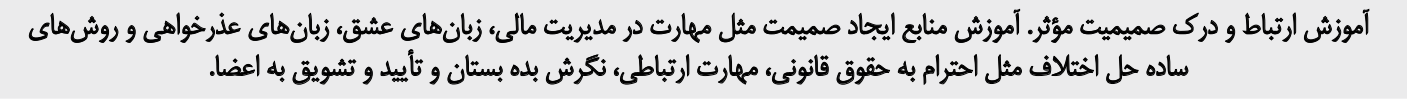 & هشتم \\
\hline 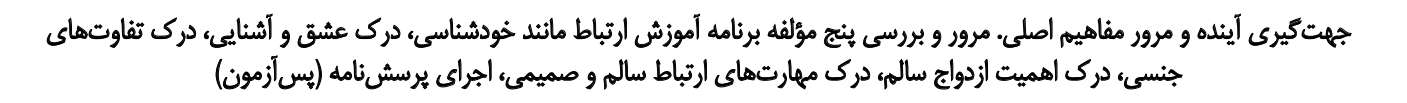 & نهم \\
\hline
\end{tabular}

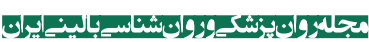

شده است. در ادامه ويرگكى هاى جمعيتشناختى و نتايج تحليل

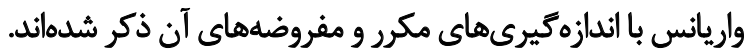

بررسى شاخصهاى جمعيتشناختى نشان داد كه ميانكين و انحراف سن كل شركت كنيندكان

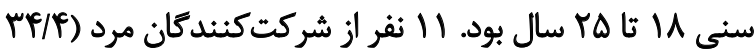

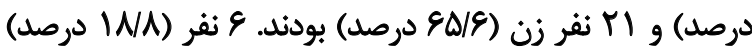

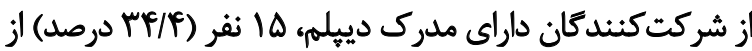

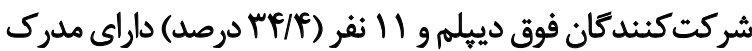

$$
\text { ليسانس و بالاتر بودند. }
$$

همانطور كه در جدول شماره r ملاحظه ميشود، ميانكين

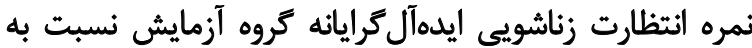

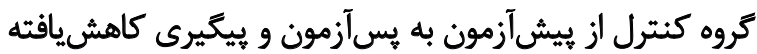

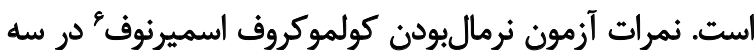

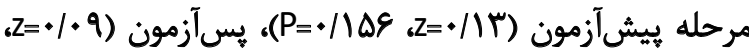
(P=•/T.

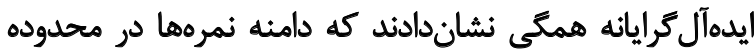

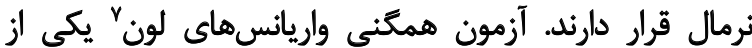

6. Kolmogorov-smirinov

7. Levene's Homogeneity of Variances Test [rإ] به دست آمده است. نيلفروشان و همكاران در مطالعهاي

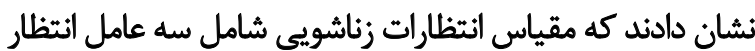

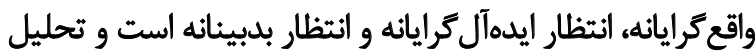

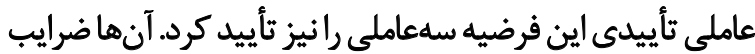

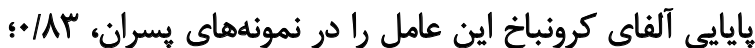

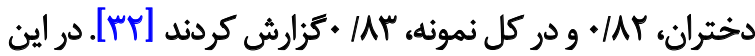

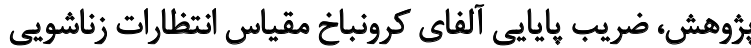

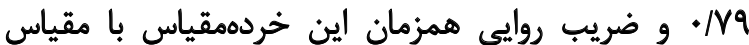

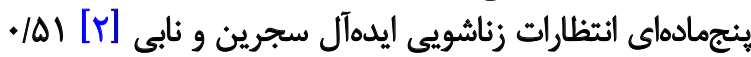

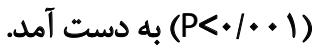

تحليل دادهها با استفاده از روشهاى آمار توصيفى مانند

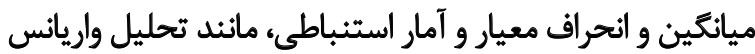

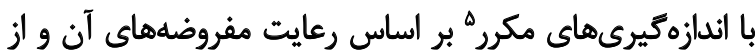

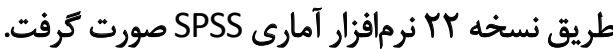

بائهنها

در اين بخش، نتايج تحليل دادهها جهت بررسى اثربخشى إنيا

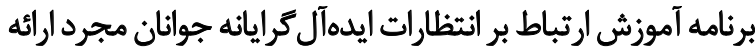

5. Repeated measures analysis of variance 
جدول r. ميانكين و انحراف معيار نمره بُعد انتظارت زناشويى ايدهآل كرايانه در مراحل يبش آزمون، يس آزمون و ييكيرى در كروههاى آزمايش و كنترل

\begin{tabular}{|c|c|c|c|}
\hline \multicolumn{3}{|c|}{ مياتكين士|نحراف معيار } & \multirow{2}{*}{ مرحله } \\
\hline ييكيرى & يَسأزمون & بيشآزمون & \\
\hline$F / A I \pm F / M r$ & $r N|I \pm V| s \mid$ & $\Delta \cdot / \varphi \Gamma \pm \Delta / .9$ & أزمايش \\
\hline 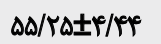 & $\Delta \Delta / A \mid \pm \Delta / \Delta$ & 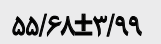 & كتترل \\
\hline
\end{tabular}

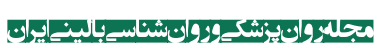

جدول ب. نتايج مقايسه تحليل واريانس تروههاى آزمايش و كنترل در بُعد انتظارت زناشويع ايدهآل ترايانه

\begin{tabular}{|c|c|c|c|c|c|}
\hline Pطح P P & Fسبت F F F F & ميانكين مجموع مجذورات & درجات آزادى & مجموع مجذورات & منبع تغييرات \\
\hline \multirow[t]{2}{*}{$<+1 . \cdot 1$} & $11 \% / / Y$ & rpar/.q & 1 & mear/.q & بين أزمودثىها: كروه \\
\hline & & $r \cdot / \Lambda \Delta$ & r. & QTW/GP & خطا \\
\hline
\end{tabular}

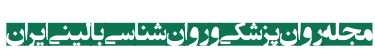

جدول F. نتايج تحليل واريانس با اندازهيرى مكرر در مراحل سهكَانه مداخله آموزشى در بُعد انتظارت زناشويى ايدهآل كرايانه

\begin{tabular}{|c|c|c|c|c|c|}
\hline Pطح P P P & Fسبت F F F F & ميانكين مجموع & درجات آزادى & مجموع مجذورات & منبع تغييرات \\
\hline$<+1+.1$ & $11 / 2 v$ & mIr/.1 & r & SYS/.Y & 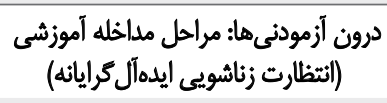 \\
\hline \multirow[t]{2}{*}{$<* / * 1$} & $11 / 41$ & MIN/Fe & r & sTr/ar & كروهها × مراحل مداخله آموزشى \\
\hline & & TW/TA & 8. & $\mid \mathrm{gTV} / . \mathrm{F}$ & خطا \\
\hline
\end{tabular}

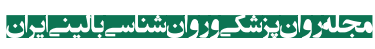

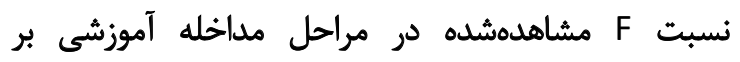

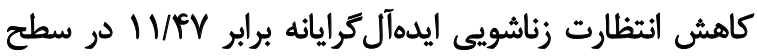

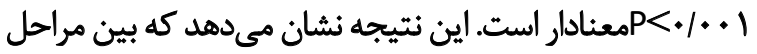

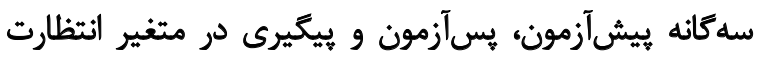

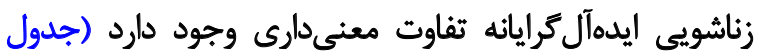

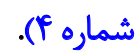

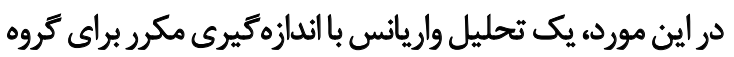

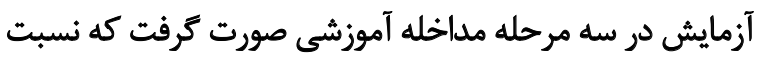

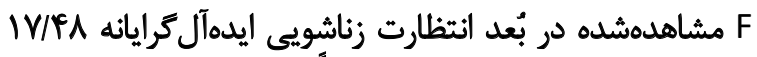

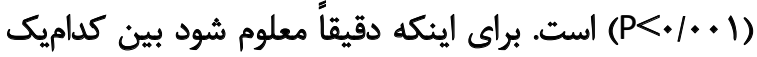

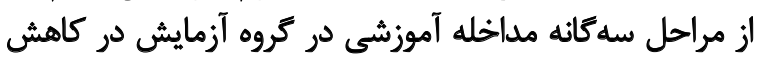

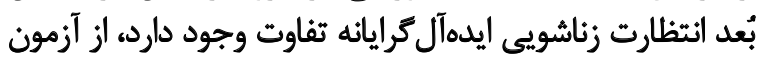
مقايسه بنفرونى • استفاده شد.

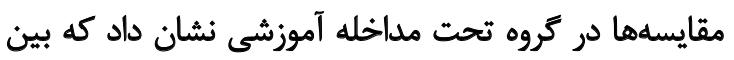

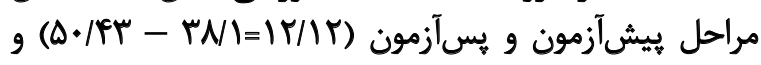

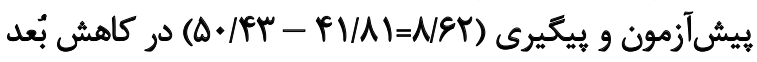

10. Bonferroni

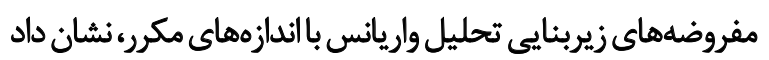

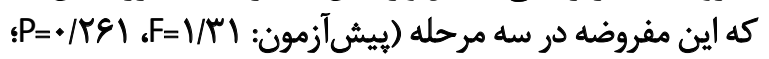

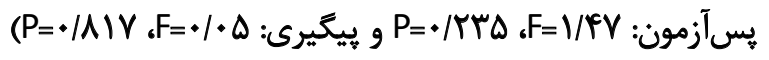

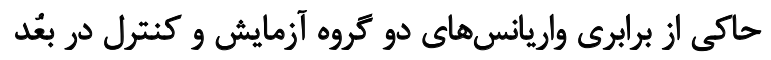

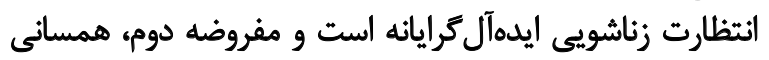

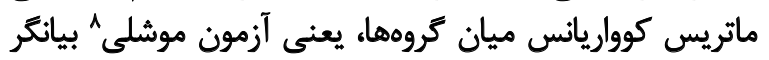

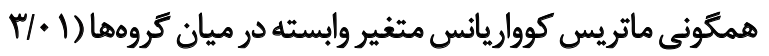

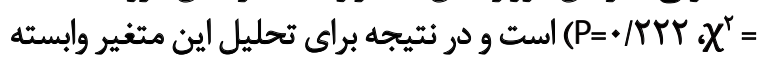

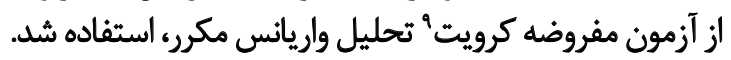

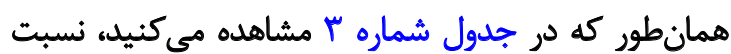

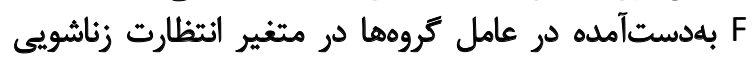

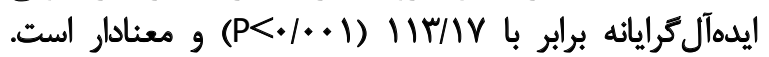

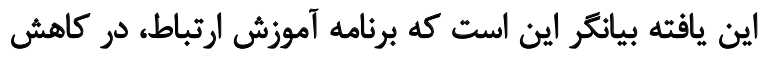

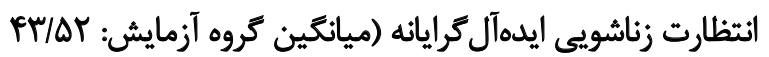
و ميانكين كروه كنترل: كنترل مؤثر است.

8. Mauchlys test

9. Sphericity assumed 


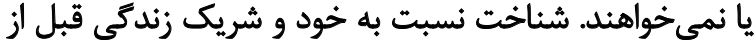

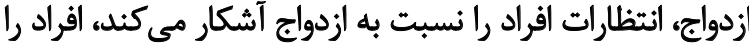

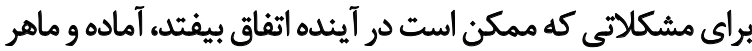

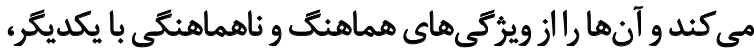

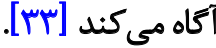

بر اين اساس يكى از مؤلفههاى برنامه آموزش ارتباط، آموزش آمان

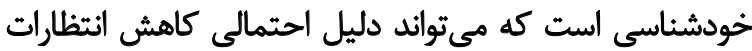

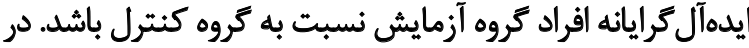

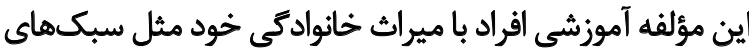

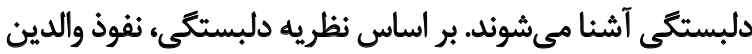

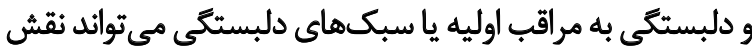

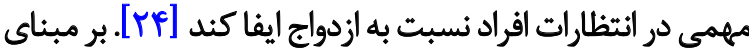

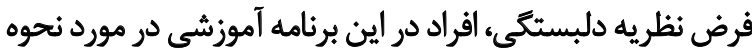

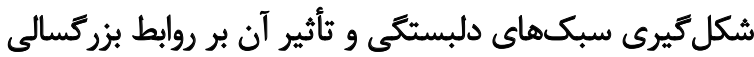

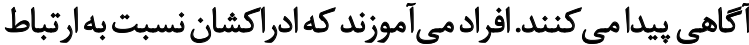

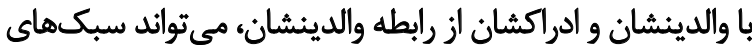

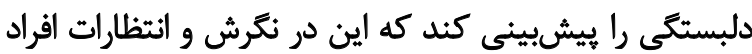

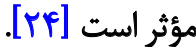

يكى ديكَر از دلايل احتمالى كاهش انتظارات ايدهآل كرايانه

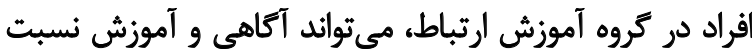

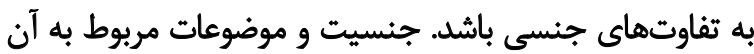

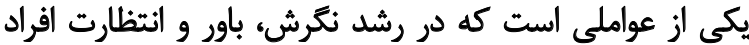

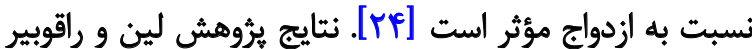

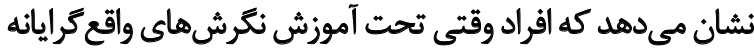

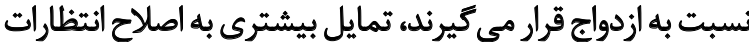

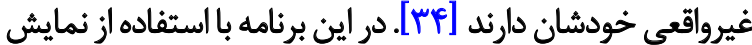

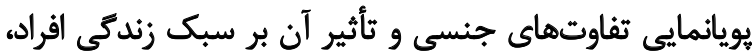

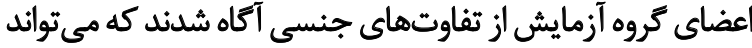

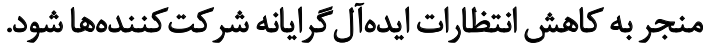

انتظارات غيرواقعى در مورد ازدواج مي تواند يكى از دلايل زندكى

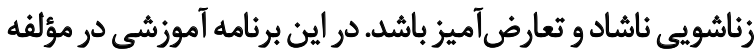

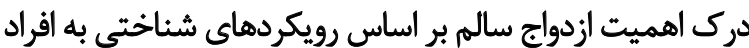

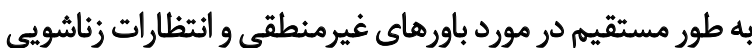

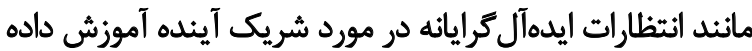

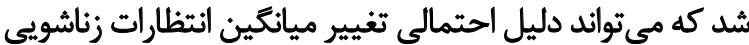

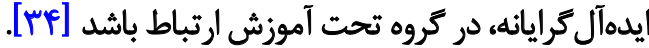

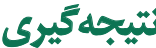

اقراد با انتظارات و عقايد غيرمنطقى، ايدهآل كرايانه و عاشقانه

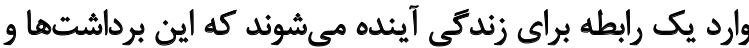

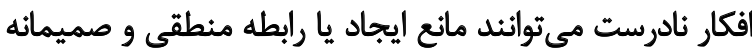

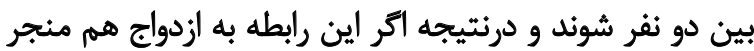

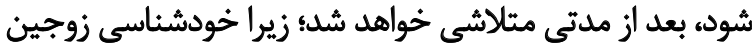

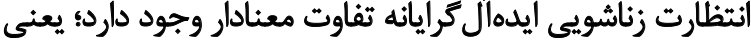

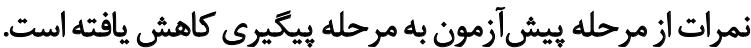

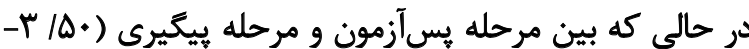

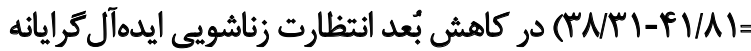

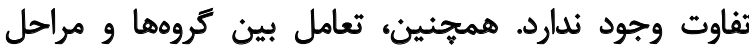

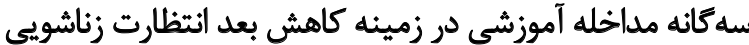

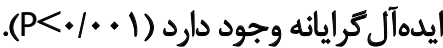

بحث

هدف اين ثيروهش بررسى اثربخشى آموزش قبل از ازدواج

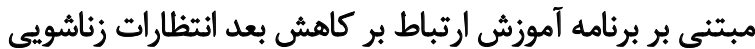

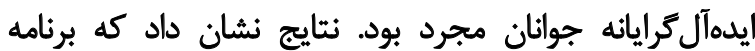

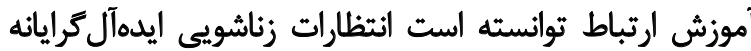

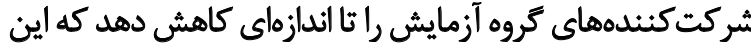

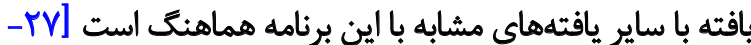

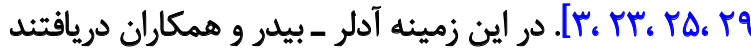

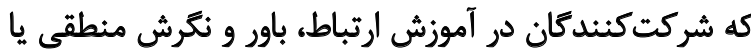

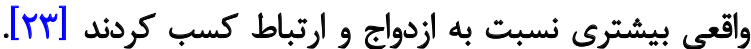

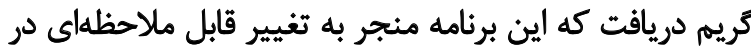

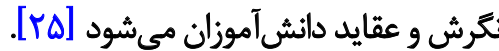

كاردنر و همكاران نيز دريافتند كه برنامهنهاى آموزش

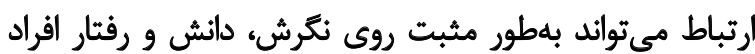

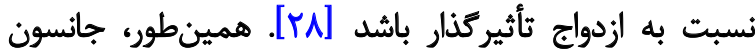
دريافت كه جوانانى كه در كلاسهاى آموني

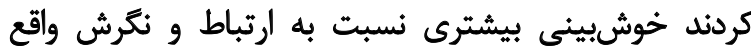

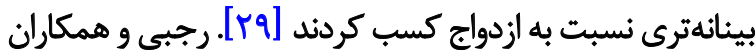

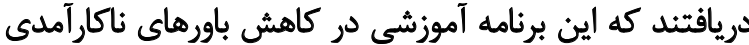

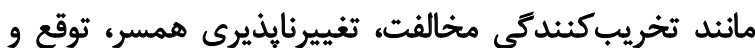

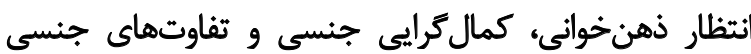

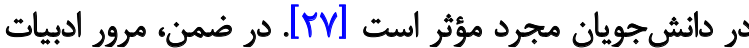
يروهش يافتههاى متضادى را نشان نداده است.

اين كونه مي توان بيان كرد كه برنامه آموزش ارتباط شامل ينج

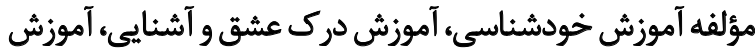

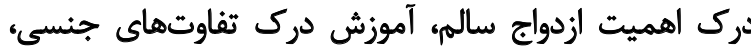

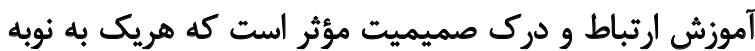

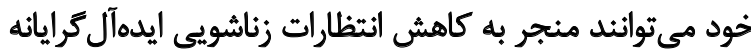

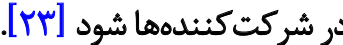

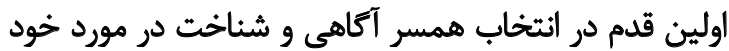

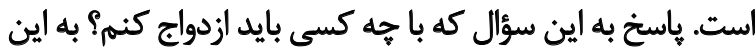

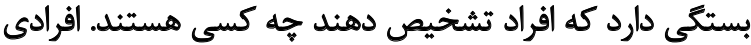

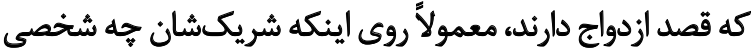

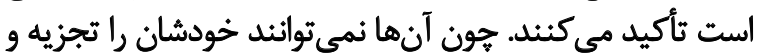

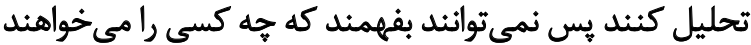




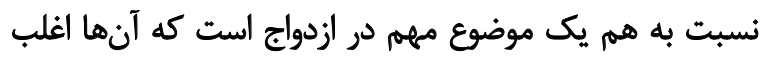

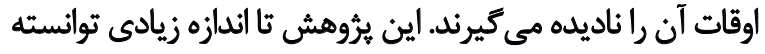

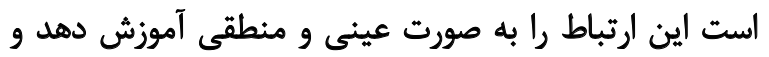

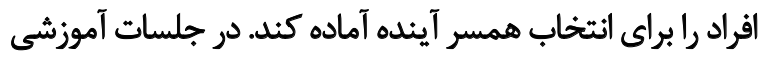

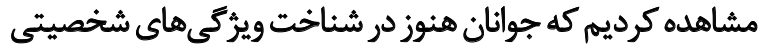

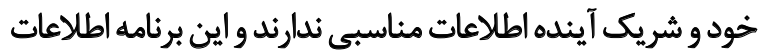
مفيدى را در مسير زندگى به آنها آنها داد.

محدوديت اين يروهش، نمونه جوانان مجرد شهر اهواز است.

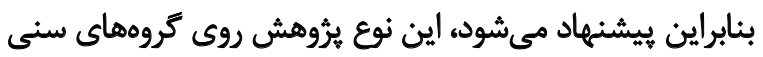

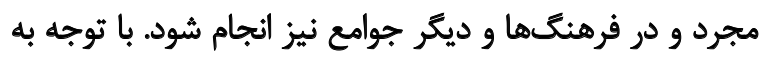

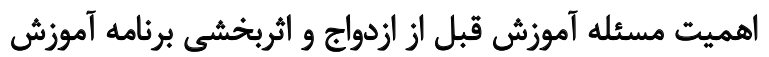

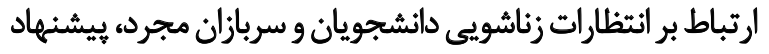

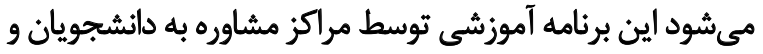

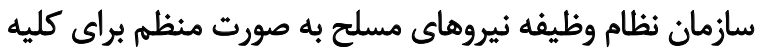

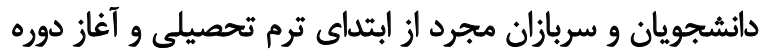

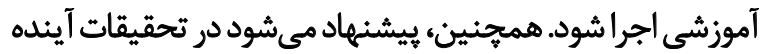

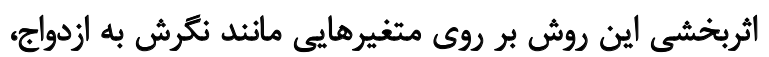
طلاق و آمادكى براى ازدواج نيز مورد بررسى قرئ مرار كيرد.

مالاحظات اخلاقي

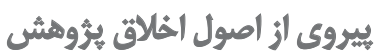

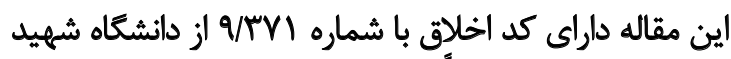

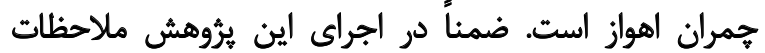

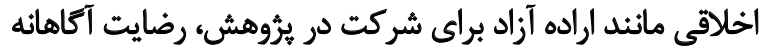

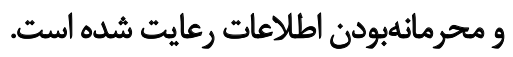

$$
\text { مائ مالى } 1 \text { اين مقاله حمايت مالى نداشته است. }
$$

تمام نويسندكان در آمادهسازى اين مقاله مشاركت داشتهاند.

$$
\text { تعارض مناقع }
$$

بنا به اظهار نويسنده مسئول مقاله، در اين يُؤوش تعارض منافع وجود نداشته است. 


\section{References}

[1] Azzopardi C. Expectations of marriage before \& after marriage among Maltese Catholic couples. [PhD. dissertation]. London: University of East London; 2007.

[2] Segrin C, Nabi RL. Does television viewing cultivate unrealistic expectations about marriage? Journal of Communication. 2002; 52(2):247-63. [DOI:10.1111/j.1460-2466.2002.tb02543.x]

[3] Rajabi Gh, Abbasi Gh, Sudani M, Aslani Kh. [Premarital education program based on premarital interpersonal choices and knowledge program on idealistic marital expectation in single Iranian students (Persian)]. Journal of Psychiatry \& Clinical Psychology. 2216; 2(3):212-21. [DOI:10.18869/acadpub.ijpcp.22.3.212]

[4] Ufholz KE. The effect of peers on marital beliefs and expectations [PhD. dissertation]. Dayton: The University of Dayton; 2012.

[5] Fletcher GJ, Simpson JA, Thomas G. Ideals, perceptions, and evaluations in early relationship development. Journal Personal Social Psychology. 2000; 79(6):933-40.

[6] Sharp EA, Ganong LH. Raising awareness about marital expectations: Are unrealistic beliefs changed by integrative teaching? Family Relations. 2000; 49(1):71-6. [DOI:10.1111/j.17413729.2000.00071.x]

[7] Rios CM. The relationship between premarital Advice, expectations and marital satisfaction [MSc. thesis]. Utah: Utah State University; 2010.

[8] Faubert K. This isn't a fairy tale: An exploration of marital expectation and coping among marriage women [MSc. thesis].Miami: Miami University; 2008.

[9] Larson JH. The marriage quiz: College students' beliefs in selected myths about marriage. Family Relationship. 1988; 37(1):3-11. [DOI:10.2307/584422]

[10] Casad BJ, Salazar MM, Macina V. The real versus the ideal: Predicting Relationship satisfaction and well-being from endorsement of marriage myths and benevolent sexism often associated with unrealistic expectations and fairy-tale. Psychology of Women Quarterly. 2014; 39(1):1-11. [DOI:10.1177/0361684314528304]

[11] Pashib M, Moharrami A, Mohammadi S, Tatari M. The effect of premarital counseling on the marriage expectation among Students University of Medical Science. Journal of Health Chime. 2016; 4(4):10-15

[12] Dillon HN. Family violence and divorce: Effects on marriage expectations [MA. thesis]. Tennessee: East Tennessee State University; 2005.

[13] Torppa CB. Preparing adolescents and young adults for marriage: Developing realistic expectations for family communication. Fact sheet family and consumer sciences. Ohio: The Ohio State University; 2009.

[14] Van Epp MC. An exploration of the dating attitudes, beliefs and behavior of single army soldiers and their perceive readiness to marry [MSc. thesis]. Ohio: The Ohio State University; 2006.

[15] Knutson L, Olson DH. Effectiveness of PREPARE program with pre-marital couples counseling in community settings. Marriage \& Family: A Christian Journal. 2003; 6(4):529-46.

[16] Valiente CE, Belanger CJ, Estrada AU. Helpful and harmful expectations of premarital interventions. Journal of Sex \& Marital Therapy. 2002; 28(1):71-7. [DOI:10.1080/009262302317251034]
[17] Hawkins AJ, Carroll JS, Doherty WJ, Willoughby B. A comprehensive framework for marriage education. Family Relations. 2004; 53(5):547-58. [DOI:10.1111/j.0197-6664.2004.00064.x]

[18] Brotherson SE, Duncan WC. Rebinding the ties that bind: Government efforts to preserve and promote marriage. Family Relations. 2004; 53(5):459-68. [DOI:10.1111/j.0197-6664.2004.00054.x]

[19] Poley JM. A pre-marriage proposal: Getting ready for marriage, an Adlerian design [MA. thesis]. Adlerian Counseling and Psychotherapy; 2011.

[20] Jamshidian Naini Y, Younesi F, Ghaderi Z. [The effectiveness of premarital skills training on the personal growth of the single girls (Persian)]. Quarterly of Cultural Counseling and Psychotherapy. 2015;3(23):121-38.

[21] Fatehi, Khashkonab, F, Karimi Thani P. [Effectiveness of premarital skills training on improving social skills and self-efficacy of girls on the brink of marriage sponsored by Imam Khomein Relief Committee of Karaj (Persian)]. Woman and Family Studies. 2015; 8(33):141-62

[22] Nielsen A, Pinsof W, Rampage C, Solomon AH, Goldstein S. Marriage 101: An integrated academic and experiential undergraduate marriage education course. Family Relations. 2004; 53(5):485-94. [DOI:10.1111/j.0197-6664.2004.00057.x]

[23] Adler-Baeder F, Kerpelman JL, Schramm DG, Higginbotham B, Paulk A. The impact of relationship education on adolescents of diverse backgrounds. Family Relations. 2007; 56(3):291-303. [DOI:10.1111/j.1741-3729.2007.00460.x]

[24] Johnson VI. The effects of intimate relationship education on relationship optimism and attitudes toward marriage $[\mathrm{PhD}$. dissertation]. Missoula, Montana: The University of Montana; 2009.

[25] Grimm AJ. Go for the gold: Relationship education program evaluation [MSc. thesis]. Dayton, Ohio: University of Dayton 2011.

[26] Halpern-Meekin S. High school relationship and marriage education: A comparison of mandated and self-Selected treatment. Journal of Family Issues. 2011; 32(3):394-419. [DOI:10.1177/0192513X10383944]

[27] Rajabi Gh, Abbasi Gh, Sudani M, Aslani Kh. [The effectiveness of premarital education based on relationship education program on reducing the dysfunctional relationship beliefs of single students (Persian)]. Journal of Applied Psychological Research. 2018 9(2):1-15.

[28] Gardner SP, Giese K, Parrot SM. Evaluation of the "connections: Relationships and Marriage" curriculum. Family Relations. 2004; 53(5):521-7. [DOI:10.1111/j.0197-6664.2004.00061.x]

[29] Johnson VI. Adult children of divorce and relationship education: Implications for counselors and counselor educators. The Family Journal: Counseling and Therapy for Couples and Families. 2011; 19(1):22-9. [DOI:10.1177/1066480710387494]

[30] Pearson M. Love U2: Getting smarter about relationships, Berkeley, CA: The Dibble Fund for Marriage Education; 2004

[31] Jones GD, Nelson ES. Expectations of marriage among college students from intact and bit-intact homes. Journal of Divorce \& Remarriage. 1996; 26(2):171-89. [DOI:10.1300/J087v26n01_09]

[32] Nilforushan P, Abedi A, Navidian A, Ahmadi SA. Studying the factor structure, reliability, and validity of the Marriage Expectation Scale (MES). Journal of Behavioral Sciences. 2011; 5(1):11-9. 
[33] Yilmaz T, Kalkan M. The effects of a premarital relationship enrichment program on relationship satisfaction. Educational Sciences: Theory and Practice. 2010; 10(3):1911-20.

[34] Lin YC, Raghubir P. Gender differences in unrealistic optimism about marriage and divorce: Are men more realistic and women more realistic? Personality and Social Psychology Bulletin. 2005; 31(2):198-207. [DOI:10.1177/0146167204271325] [PMID] 